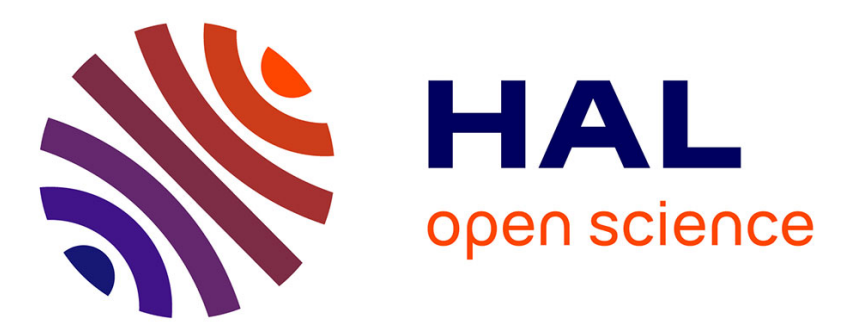

\title{
Convergence of poroelastic finite elements based on Biot displacement formulation
}

Nicolas Dauchez, Sohbi Sahraoui, Noureddine Atalla

\section{To cite this version:}

Nicolas Dauchez, Sohbi Sahraoui, Noureddine Atalla. Convergence of poroelastic finite elements based on Biot displacement formulation. Journal of the Acoustical Society of America, 2001, 109 (1), pp.3340. 10.1121/1.1289924. hal-00438864

\section{HAL Id: hal-00438864 https://hal.science/hal-00438864}

Submitted on 31 Dec 2009

HAL is a multi-disciplinary open access archive for the deposit and dissemination of scientific research documents, whether they are published or not. The documents may come from teaching and research institutions in France or abroad, or from public or private research centers.
L'archive ouverte pluridisciplinaire HAL, est destinée au dépôt et à la diffusion de documents scientifiques de niveau recherche, publiés ou non, émanant des établissements d'enseignement et de recherche français ou étrangers, des laboratoires publics ou privés. 


\title{
Convergence of poroelastic finite elements based on Biot displacement formulation
}

\author{
Nicolas Dauchez and Sohbi Sahraoui \\ Laboratoire d'Acoustique UMR CNRS 6613, Univ. du Maine, \\ 72095 Le Mans cedex 9, France \\ Noureddine ATALLA \\ GAUS, Mechanical Engineering Dpt, Université de Sherbrooke, \\ Sherbrooke, Québec, J1K 2R1, Canada
}

Revised manuscript (14 May 2000)

Shortened title : Convergence of Biot finite elements

Nicolas Dauchez, Sohbi Sahraoui, Noureddine Atalla

The Journal of the Acoustical Society of America 


\section{ABSTRACT}

The convergence of linear poroelastic elements based on Biot displacement formulation is investigated. The aim is to determine a mesh criterion that provides reliable results under a given frequency limit. The first part deals with 1-D applications for which resonance frequencies can be related to Biot wavelengths. Their relative contributions to the motion are given in order to determine the mesh criteria for monophasic media for poroelastic media. The imposition of 6 linear elements per wavelength is found for each Biot wave as a primary condition for convergence. For 3-D applications, convergence rules are derived from a generic configuration, i.e. a clamped porous layer. Because of the complex deformation, previous criterion is shown to be insufficient. Influence of the coupling between the two phases is demonstrated.

PACS numbers : 43.20.Jr, 43.50.Gf, 43.40.Tm, 43.55.Wk

Nicolas Dauchez, Sohbi Sahraoui, Noureddine Atalla

The Journal of the Acoustical Society of America 


\section{INTRODUCTION}

Biot theory of fluid saturated porous media provides a description of the waves propagating in geophysics materials (Biot, 1956). Several authors have extended this theory to sound absorbing materials, such as glass wool and polymer foams. A good agreement with measurements has been shown for multilayer systems using analytical methods (Allard, 1993). In order to interpret the modal behavior of finite size structures, 2-D and 3-D finite element formulations based on extended Biot theory have been developed (Coyette and Wynendaele, 1995; Johansen et al., 1995; Kang and Bolton, 1995; Panneton and Atalla, 1997). These formulations have been validated by comparison with measured or simulated surface impedance of layered media. Several authors have also performed parametric studies. However the convergence of the different formulations has not been specifically studied.

Because of the biphasic nature of poroelastic elements, a mesh criterion used for elements describing monophasic media (solid or fluid) is a priori not valid for poroelastic elements. Such a criterion is essential for designing a suitable mesh that gives reliable results. Moreover, an optimized mesh will result in an important saving of computer time and memory, as poroelastic formulation leads to heavy computation loads. Determination of convergence criterion for poroelastic elements is an objective of this paper.

The finite element formulation used in this study is based on Biot-Johnson-Allard theory (Allard, 1993) and has been implemented by Panneton and Atalla (1997). It is referred to as the $(u, U)$ formulation for solid and fluid displacements. Poroelastic elements have 6 degrees of freedom per node ( 3 translations for each phase). They are 
based on linear three-dimensional pentaedric or hexaedric elements.

The first part deals with 1-D applications for which resonance frequencies can be related to Biot waves. Their relative contributions to the motion are investigated in order to determine whether criteria used for monophasic media are suitable for poroelastic media.

The second part deals with convergence of 3-D applications where the finite size of the structure is relevant. In that case, the displacement of both phases can be fairly different and the relation between resonances and wavelengths is no longer direct. Convergence rules are derived from a generic configuration, i.e. a clamped porous layer.

\section{CONVERGENCE FOR 1-D BEHAVIOR}

\section{A. Theoretical analysis}

The motion of the poroelastic media is described by the macroscopic displacement of the solid and fluid phases represented by the vectors $\boldsymbol{u}$ and $\boldsymbol{U}$. Assuming a harmonic time dependence $e^{j \omega t}$, the equation of motion can be written in the form (Allard, 1993)

$$
\begin{array}{r}
P \nabla(\boldsymbol{\nabla} \cdot \boldsymbol{u})-N \boldsymbol{\nabla} \wedge(\boldsymbol{\nabla} \wedge \boldsymbol{u})+Q \boldsymbol{\nabla}(\boldsymbol{\nabla} \cdot \boldsymbol{U})+\omega^{2}\left(\tilde{\rho}_{11} \boldsymbol{u}+\tilde{\rho}_{12} \boldsymbol{U}\right)=0 \\
Q \boldsymbol{\nabla}(\boldsymbol{\nabla} \cdot \boldsymbol{u})+R \boldsymbol{\nabla}(\boldsymbol{\nabla} \cdot \boldsymbol{U})+\omega^{2}\left(\tilde{\rho}_{12} \boldsymbol{u}+\tilde{\rho}_{22} \boldsymbol{U}\right)=0
\end{array}
$$

where $P$ and $R$ are the bulk modulus of the solid and fluid phases respectively, $N$ is the shear modulus of the solid phase and $Q$ quantifies the potential coupling between the two phases. These coefficients are complex : $P, N$ include structural damping in the solid phase and $R, Q$ thermal dissipation. The equivalent mass density coefficients $\tilde{\rho}_{11}, \tilde{\rho}_{12}, \tilde{\rho}_{22}$ account for inertial and viscous coupling between both the solid and fluid phases.

Nicolas Dauchez, Sohbi Sahraoui, Noureddine Atalla

The Journal of the Acoustical Society of America 
Coupling between the two phases is described by 5 parameters according to JohnsonAllard theory (Allard, 1993) : $\phi$ porosity, $\alpha_{\infty}$ tortuosity, $\sigma$ air flow resistivity, $\Lambda$ and $\Lambda^{\prime}$ viscous and thermal characteristic lengths respectively.

\section{Properties of Biot waves}

As dilatational and rotational waves are uncoupled, the equation of motion can be solved separately for the two types of wave. Scalar potentials for dilatation and vector potentials for shear has been used by Biot to express the displacement of the solid and fluid phases. Two compressional waves characterized by their constant of propagation $k_{1}$ and $k_{2}$ are found

$$
\begin{aligned}
& k_{1}^{2}=\frac{\omega^{2}}{2\left(P R-Q^{2}\right)}\left(P \tilde{\rho}_{22}+R \tilde{\rho}_{11}-2 Q \tilde{\rho}_{12}-\sqrt{\Delta}\right), \\
& k_{2}^{2}=\frac{\omega^{2}}{2\left(P R-Q^{2}\right)}\left(P \tilde{\rho}_{22}+R \tilde{\rho}_{11}-2 Q \tilde{\rho}_{12}+\sqrt{\Delta}\right),
\end{aligned}
$$

with

$$
\Delta=\left(P \tilde{\rho}_{22}+R \tilde{\rho}_{11}-2 Q \tilde{\rho}_{12}\right)^{2}-4\left(P R-Q^{2}\right)\left(\tilde{\rho}_{11} \tilde{\rho}_{22}-\tilde{\rho}_{12}^{2}\right)
$$

Each wave propagates in both phases with different amplitudes. The ratio $\mu_{i}$ between displacement of fluid and solid phases, respectively denoted $U_{i}$ and $u_{i}$ for each wave $i$, is given by

$$
\mu_{i}=\frac{U_{i}}{u_{i}}=\frac{P k_{i}^{2}-\omega^{2} \tilde{\rho}_{11}}{\omega^{2} \tilde{\rho}_{12}-Q k_{i}^{2}}, \quad i=1,2
$$

Following the nomenclature used by Allard (1993), the wave that propagates mainly in the fluid is called the fluid-borne wave, and the one that propagates mainly in the solid is called the solid-borne wave. This nomenclature reflects the physical nature of the two waves for acoustical porous materials at low frequencies. The solid-borne wave 
propagates in the two phases with similar amplitude. The fluid-borne wave propagates mainly in the fluid phase, and is usually strongly damped. Each kind of wave will be identified by over-script $s w$ or $f w$, respectively for solid-borne and fluid-borne wave. Note that each wave has not always the same $k_{i}$ over the whole frequency range.

One shear wave propagates in the porous medium, characterized by

$$
k_{3}^{2}=\frac{\omega^{2}}{N}\left(\frac{\tilde{\rho}_{11} \tilde{\rho}_{22}-\tilde{\rho}_{12}^{2}}{\tilde{\rho}_{22}}\right) \quad \text { and } \quad \mu_{3}=-\frac{\tilde{\rho}_{12}}{\tilde{\rho}_{22}}
$$

For each Biot wave $i$, celerity $c_{i}$, wavelength $\lambda_{i}$ and wave damping $\gamma_{i}$ are deduced from the following relation,

$$
k_{i}=\frac{\omega}{c_{i}}-j \gamma_{i}=\frac{2 \pi}{\lambda_{i}}-j \gamma_{i} \quad i=1,2,3
$$

\section{Relative contributions of longitudinal Biot waves}

Consider a porous layer of thickness $l$ along the $x$ axis (figure 1 ). The displacement of one fluid or solid particle is the contribution of four waves : two waves ( $s w$ and $f w$ ) propagating in the $x$ direction, noted by subscript + , and two waves propagating in the $-x$ direction, noted - . Displacement of solid phase $u(x)$ and fluid phase $U(x)$ of coordinate $x$ is given by

$$
\begin{aligned}
u(x)= & u_{+}^{s w}(0) e^{-j k^{s w} x}+u_{-}^{s w}(0) e^{+j k^{s w} x} \\
& +u_{+}^{f w}(0) e^{-j k^{f w} x}+u_{-}^{f w}(0) e^{+j k^{f w} x}, \\
U(x)= & \mu^{f w}\left(u_{+}^{f w}(0) e^{-j k^{f w} x}+u_{-}^{f w}(0) e^{+j k^{f w} x}\right) \\
& +\mu^{s w}\left(u_{+}^{s w}(0) e^{-j k^{s w} x}+u_{-}^{s w}(0) e^{+j k^{s w} x}\right),
\end{aligned}
$$

where the fluid displacements have been eliminated by using the ratios $\mu^{s w}$ and $\mu^{f w}$. 
Acoustical excitation. The porous layer is first supposed to be bonded to a rigid impervious wall (figure 1 ) at $x=0$, which gives

$$
u_{+}^{s w}(0)=-u_{-}^{s w}(0), \quad u_{+}^{f w}(0)=-u_{-}^{f w}(0) .
$$

The excitation is an incident plane wave imposing a pressure $p$ at $x=-l$. The stress equilibrium at the surface of the layer is given by

$$
\begin{aligned}
-(1-\phi) p(-l) & =\sigma_{x x}^{s}(-l) \\
& =P \frac{\partial u(-l)}{\partial x}+Q \frac{\partial U(-l)}{\partial x}, \\
-\phi p(-l) & =\sigma_{x x}^{f}(-l) \\
& =Q \frac{\partial u(-l)}{\partial x}+R \frac{\partial U(-l)}{\partial x},
\end{aligned}
$$

where $\sigma_{x x}^{s}$ and $\sigma_{x x}^{f}$ are respectively the stress in the solid and fluid phases. From equations (8-12), one can calculate $u_{+}^{s w}(0)$ and $u_{+}^{f w}(0)$. Displacements at any position $x$ are given by

$$
\begin{gathered}
u(x)=u_{+}^{s w}(0)\left[2 j \sin \left(-k^{s w} x\right)+\frac{u_{+}^{f w}(0)}{u_{+}^{s w}(0)} 2 j \sin \left(-k^{f w} x\right)\right], \\
\left.U(x)=u_{+}^{f w}(0)\left[2 j \mu^{f w} \sin \left(-k^{f w} x\right)\right)+\frac{u_{+}^{s w}(0)}{u_{+}^{f w}(0)} \mu^{s w} 2 j \sin \left(-k^{s w} x\right)\right],
\end{gathered}
$$

with

$$
\frac{u_{+}^{f w}(0)}{u_{+}^{s w}(0)}=-\frac{\phi\left(P+\mu^{s w} Q\right)-(1-\phi)\left(Q+\mu^{s w} R\right)}{\phi\left(P+\mu^{f w} Q\right)-(1-\phi)\left(Q+\mu^{f w} R\right)} \times \frac{k^{s w} \cos \left(k^{s w} l\right)}{k^{f w} \cos \left(k^{f w} l\right)}
$$

Equations $(13,14)$ can be rewritten in the form

$$
\begin{gathered}
u(x)=u_{+}^{s w}(0)\left[C_{s w / s}(x)+C_{f w / s}(x)\right], \\
U(x)=u_{+}^{f w}(0)\left[C_{f w / f}(x)+C_{s w / f}(x)\right],
\end{gathered}
$$

Nicolas Dauchez, Sohbi Sahraoui, Noureddine Atalla 
where $C_{a w / b}(x)$ are contributions of a kind $a w$ of wave on the motion of one phase $b(a$ or $b$ being $s$ for solid or $f$ for fluid) at the abscissa $x$. Relative contributions are defined by setting that $\left|C_{s w / b}(x)\right|+\left|C_{f w / b}(x)\right|=100 \%$.

Figure 2 gives an example of calculation of relative contributions $\left|C_{s w / f}\right|$ and $\left|C_{f w / s}\right|$ at the incident face, $x=-l$, for a $10 \mathrm{~cm}$ thick layer of material A (table 1 ). According to $\left|C_{s w / f}\right|$, the motion of the fluid phase is governed at $20 \%$ by the solid-borne wave up to $100 \mathrm{~Hz}$. The influence of the solid-borne wave increases strongly at the first $\frac{\lambda}{4}$ resonance of the solid phase at $300 \mathrm{~Hz}$. For higher frequencies, because of decoupling, its influence tends to zero. According to $\left|C_{f w / s}\right|$, the motion of the solid phase is governed at $50 \%$ by the fluid-borne wave up to $100 \mathrm{~Hz}$. But the influence of the fluid-borne wave decreases to $20 \%$ at the first resonance of the solid phase. For higher frequencies, it tends to an asymptotic value of $65 \%$ approximately. Except around the first resonance of the solid phase, where the contribution of the fluid-borne wave decreases strongly, both waves must be carefully represented by the finite element discretization.

One can notice that when the solid phase becomes stiffer, $\mu_{s w}$ (equation (5)) increases and fluid-borne wave governs the motion of the two phases. The solid phase tends to be motionless and the behavior of the porous material can be described by an equivalent fluid model (Allard, 1993).

Imposed displacement. In this case, the same displacement is imposed on the phases at the incident face : $u(-l)=U(-l)$. If the layer is bonded to a rigid wall at $x=0$, equations (13) and (14) apply and the coefficients $C_{f w / s}(-l), C_{s w / f}(-l)$ are determined using

$$
\frac{u_{+}^{f w}(0)}{u_{+}^{s w}(0)}=-\frac{1-\mu^{s w}}{1-\mu^{f w}} \frac{\sin ^{s w} l}{\sin ^{f w} l}
$$


Because $\mu^{s w}$ is usually very close to 1 and $\mu^{f w}$ is much greater than $\mu^{s w}$, one find $\left|C_{f w / s}(-l)\right|$ close to 0 and $\left|C_{s w / f}(-l)\right|$ very large. This means that the solid-borne wave governs the motion of the two phases. The criterion would then be related only to this wave. The same conclusion arises if the back face of the layer is free or if the layer has an infinite thickness.

\section{Theoretical convergence rate}

For purpose of comparison, theoretical convergence rate has been calculated for a monophasic clamped-free rod of length $l$, discretized by 1-D linear elements (see appendix for details). Figure 3 give the relative error of the calculation of natural frequencies as a function of number of elements per wavelength. Mesh criterion is then defined as follows : with 6 linear elements per wavelength, the overestimation of natural frequencies is less than $5 \%$.

\section{B. Validation of mesh criterion}

In this part, relation between Biot wavelengths and convergence rate is verified for various configurations and 3 porous materials. The material properties (table 1) are chosen so that different behaviors according to the waves characteristics (wavelengths given in figure 11) are represented :

- material A is a standard polymer foam for which wavelength of the two dilatational waves are of the same order $\left(\lambda_{s w}=0.61 \mathrm{~m}, \lambda_{f w}=0.51 \mathrm{~m}\right.$ at $\left.200 \mathrm{~Hz}\right)$,

- material B is an over-tortuous material so that the fluid-borne wave is much shorter than the solid-borne wave $\left(\lambda_{s w}=1.24 \mathrm{~m}, \lambda_{f w}=0.05 \mathrm{~m}\right.$, at $\left.200 \mathrm{~Hz}\right)$, 
- material $\mathrm{C}$ is an intermediate material $\left(\lambda_{s w}=1.00 \mathrm{~m}, \lambda_{f w}=0.42 \mathrm{~m}\right.$ at $\left.200 \mathrm{~Hz}\right)$, corresponding to a usual absorbing material.

Three kinds of excitation and boundary conditions are applied to a poroelastic column of axis $x$ (figure 4 ) in order to excite each type of wave in the most independent way :

- excitation only of the shear wave : the direction of the force is normal to axis $x$ and only displacements normal to this axis are allowed.

- excitation mainly of the solid-borne wave : the same displacement along axis $x$ is imposed on both phases, and only displacements along this axis are allowed.

- excitation of the two longitudinal waves : the column is bonded to a rigid impervious wall and only $x$ displacements are allowed elsewhere. The free face is excited by a longitudinal force $F$ distributed on the surface fraction of each phase : $F_{s}=(1-\phi) F$ and $F_{f}=\phi F$. This surface force results from a normal incidence acoustical wave.

Only two nodes are used in the lateral directions and excitation is applied uniformly to get a 1-D behavior.

For each configuration, we first verify the relation between converged resonance frequencies and theoretical wavelengths. Converged resonance frequencies are obtained with a great number of elements in the $x$ direction. Then relative errors on resonance frequencies obtained with coarse meshes are given versus number of elements per wavelength. The frequency range of interest is approximately between $10 \mathrm{~Hz}$ and $2000 \mathrm{~Hz}$, in order to observe at least the two first resonances. 


\section{Simple shear displacement}

Figure 5.a shows three resonances at $257 \mathrm{~Hz}, 768 \mathrm{~Hz}$ and $1288 \mathrm{~Hz}$, for a $10 \mathrm{~cm}$ thick column of material C. Both phases have almost the same motion at low frequencies. The curves separates slightly as the frequency increases due to progressive decoupling.

Shear wavelengths are $0.399 \mathrm{~m}$ at $257 \mathrm{~Hz}, 0.134 \mathrm{~m}$ at $768 \mathrm{~Hz}$ and $0.080 \mathrm{~m}$ at $1288 \mathrm{~Hz}$. They are related respectively to a $\frac{\lambda}{4}, \frac{3}{4} \lambda$ and $\frac{5}{4} \lambda$ resonance.

Figure 5.b shows that convergence follows the theoretical rate (figure 3) when the number of elements is related to the shear wavelength : the criterion is suitable in this case. The same results are found with the other materials.

\section{Imposed longitudinal displacement}

Figure 6.a shows two resonances at $500 \mathrm{~Hz}$ and $1512 \mathrm{~Hz}$ for a $10 \mathrm{~cm}$ thick column of material C. The phases have almost the same motion according to paragraph I.A.2. The curves separates slightly as the frequency increases due to progressive decoupling. Solid-borne wavelengths are $0.4017 \mathrm{~m}$ at $500 \mathrm{~Hz}$ and $0.1335 \mathrm{~m}$ at $1512 \mathrm{~Hz}$. They are related respectively to a $\frac{\lambda}{4}$ and a $\frac{3}{4} \lambda$ resonance.

Figure 6.b presents the convergence rate for the two element shapes and two materials. The convergence follows the theoretical rate (figure 3) when the number of elements are related to the solid-borne wavelength : the criterion is verified.

\section{Acoustical excitation}

Because of very different longitudinal wavelengths, material B is best suited to distinguish phenomena. The thickness of the column is $7.5 \mathrm{~cm}$.

Figure 9.a shows a resonance at $19 \mathrm{~Hz}$ corresponding to a $\frac{\lambda}{4}$ resonance related to the 
fluid-borne wave. Its wavelength is $32 \mathrm{~cm}$ at $19 \mathrm{~Hz}$. Another resonance around $809 \mathrm{~Hz}$ corresponds to a $\frac{\lambda}{4}$ resonance related to the solid-borne wave. Its wavelength is $30.6 \mathrm{~cm}$ at $809 \mathrm{~Hz}$.

Figure 9.b presents the convergence rate where the number of elements is related to the appropriate wavelength. Convergence follows once again the theoretical rate. It is less clear for the fluid phase, because only its first resonance is noticeable and its convergence occurs between 1 and 2 elements for the whole column. Moreover, because the fluid-borne wave is strongly damped, the determination of the supposed resonance frequency is less accurate.

Because both longitudinal waves are excited, the mesh should verify the criterion for both waves.

Mesh optimization. According to paragraph I.A.2, situations can be encountered where one of the two longitudinal waves has little influence on the behavior of the porous material. If its wavelength is the longest, less elements are to be used to describe properly the motion.

For example, if criterion is applied to the $7.5 \mathrm{~cm}$ thick column of material B up to $1100 \mathrm{~Hz}, 2$ elements are required for the solid-borne wave $\left(\lambda_{s w}=22.5 \mathrm{~cm}\right)$ whereas 30 elements are required for the fluid-borne wave $\left(\lambda_{f w}=1.5 \mathrm{~cm}\right)$. However figure 8.a shows that the contribution of the fluid-borne wave decreases as long as the first resonance related to the solid-borne wave is reached : the contribution of fluid-borne wave $\left|C_{f w / s}\right|$ on the solid phase motion is less than $20 \%$ after $50 \mathrm{~Hz}$ and the contribution of solid-borne wave $\left|C_{s w / f}\right|$ on the fluid motion increases up to $80 \%$ at $809 \mathrm{~Hz}$. Consequently, with only 3 elements, the motion of the fluid phase is approximated with an error less than $3 \mathrm{~dB}$, 
as shown by figure 8.b. Above $900 \mathrm{~Hz}$, error slightly increases due to the decrease of the influence of the solid-borne wave.

Irregular mesh. The fluid-borne waves are usually strongly damped. If it corresponds to the shortest wave, a mesh reduction can be achieved by respecting criterion for this wave only in the area close to the excitation face. Elsewhere, a criterion based on the solid-borne wave will be suitable.

For example, figure 9.a shows a quick variation close to the excited face, corresponding to the fluid phase motion at $809 \mathrm{~Hz}$. This variation is directly related to the fluid-borne wave $\left(\lambda_{f w}=1.9 \mathrm{~cm}\right.$, at $\left.809 \mathrm{~Hz}\right)$ which is strongly attenuated. The best results are achieved using a mesh with 3 elements of size increasing from $0.1 \mathrm{~cm}$ to $5 \mathrm{~cm}$ (figure 9.b) rather than using 3 regular sized elements.

The choice of such an irregular mesh can be guided using equations (13) and (14) to calculate the solid and fluid phase displacements along $x$ axis.

To summarize, for acoustical excitation, criterion is applied according to both longitudinal waves, but a mesh optimization can be performed if the shortest wave is not predominant or strongly damped. If the shear wave is relevant in the motion of the poroelastic media, then the criterion has to be applied according to this wave too. In a general way, the verification of criterion according to each of the three waves is a necessary condition to insure reliable results. 


\section{CONVERGENCE FOR 3-D BEHAVIOR}

For real 3-D behavior, the contribution of the Biot waves is difficult to predict. Verification of the criterion for each wave appears to be a necessary condition. Nevertheless convergence will not necessarily follow the same rules.

We investigate now the convergence of a generic case in order to specify some convergence rules. The generic case is a clamped porous layer of material A, excited by a plane wave. The dimensions are $28 \mathrm{~cm} \times 35 \mathrm{~cm}$ and the thickness is $3 \mathrm{~cm}$. Hexaedric linear elements are used.

\section{A. Convergence of equivalent solid elements}

We first consider the behavior without coupling effects. Poroelastic elements are replaced by equivalent solid elements with the same stiffness of the frame and the density of the air filled porous material. Equivalent element is based on the same hexaedric element used for poroelastic element. The frequency of the first bending mode is given by table 2 for several meshes refined in lateral and thickness directions. The converged frequency is $48 \mathrm{~Hz}$. It is shown that within an error of $5 \%$, convergence is reached with a $2 \times 12 \times 12$ elements mesh. Related to the deformation shape, this yields almost 24 elements per wavelength : it is 4 times the 6 element per wavelength criterion.

This slow convergence can be explained by locking phenomenon of linear elements subject to bending (MacNeal, 1994). Locking induces excessive stiffness for a particular deformation state and is related primarily to interpolation failure. For bending deformation, two kinds of locking are noticed. Shear locking is related to a bad interpolation of the deflection which should be a second order polynomial. A mesh refinement in the 
in-plane direction will reduce this locking effect. Dilatation locking is due to a bad interpolation of thickness dilatation, induced by Poisson's effect, which should be a second order polynomial too. The phenomenon is more important when $\nu$ is close to 0.5 . A mesh refinement in the thickness direction will reduce this locking effect.

In order to minimize these effects, selective under-integration (MacNeal, 1994) has been performed for shear strain in our hexaedric element.

\section{B. Convergence of poroelastic elements with coupling effects}

Imposition of the 1-D criterion for the three waves requires only 2 elements in lateral directions and less than 1 element in the thickness at $50 \mathrm{~Hz}$. According to results obtained with equivalent elements, the minimal mesh should be $2 \times 12 \times 12$ elements.

Nevertheless, table 3.a shows that the resonance frequency is overestimated by $10 \%$ instead of $5 \%$ without coupling effects. Table 3.b shows that convergence of dissipated power by viscous effects $D_{c p}$ is even slower, and very sensitive to a refinement in the thickness. Moreover, when the mesh is too coarse in the thickness, convergence is not always monotonic as the mesh is refined in the lateral directions.

\section{CONCLUSION}

It has been shown that linear poroelastic elements verify the convergence rate of linear monophasic elements, according to each type of Biot wave. Classical mesh criterion, i.e. 6 linear elements per wavelength, provides a necessary condition to obtain reliable results.

For real 3-D deformations, like bending, classical criterion gives indications for a minimal mesh but is insufficient because of locking of 3-D linear elements and discrepancies of the displacement field of the two phases. A consequent refinement of the 
mesh is necessary to obtain satisfactory results. Indicators related to the fluid motion have been found very sensitive. As a consequence, the minimal number of elements required is difficult to predict.

By nature, the convergence of poroelastic elements is slower than the convergence of either equivalent solid or fluid elements, because of the presence of two different scale phenomena. The discrepancy can be lowered when the behavior of the porous material is dominated by the motion of one phase.

As a consequence, this slow convergence leads to large systems to be solved. Use of higher order elements together with the $(u, p)$ formulation (Atalla et al., 1996, 1998), that requires only 4 degrees of freedom per node, would be an efficient way to reduce the size of the systems. Another way would be to design specific models dedicated to particular applications.

\section{ACKNOWLEDGMENTS}

The authors are grateful to PSA Peugeot Citroën and Agence de l'Environnement et de la Maîtrise de l'Energie (Ademe) who have supported this study.

\section{APPENDIX : Theoretical convergence rate for 1-D monophasic linear elements}

Consider a clamped-free rod of length $l$, Young's modulus $E$ and mass per unit volume $\rho$. Assuming harmonic longitudinal motion along the $x$ axis, the mode shape $r$ is given by (Blevins, 1995)

$$
w_{r}(x)=w_{0} \sin \left(\frac{2 r-1}{l} \frac{\pi}{2} x\right)
$$

Nicolas Dauchez, Sohbi Sahraoui, Noureddine Atalla

The Journal of the Acoustical Society of America 
with natural frequencies

$$
\omega_{r}=\frac{\pi}{2} \sqrt{\frac{E}{\rho l^{2}}}(2 r-1) .
$$

Approximation with $m$ linear elements of the same size leads to a system of $m-1$ equations of the form (Géradin and Rixen, 1996)

$$
E \frac{m}{l}\left(-\bar{w}_{i-1}+2 \bar{w}_{i}-\bar{w}_{i+1}\right)-\omega^{2} \frac{l}{6 m}\left(\bar{w}_{i-1}+4 \bar{w}_{i}+\bar{w}_{i+1}\right)=0
$$

where $\bar{w}_{i}$ is the displacement of the $i^{\text {th }}$ node for $0<i<m$. With a general solution of the form

$$
\bar{w}_{i, r}=w_{0} \sin \left(i \bar{k}_{r}+\theta\right)
$$

we get wavenumbers and approximated natural frequencies

$$
\begin{gathered}
\bar{k}_{r}=\frac{2 r-1}{m} \frac{\pi}{2} \\
\bar{\omega}_{r}=\frac{1}{\sqrt{3}} \sqrt{\frac{E}{\rho l^{2}}} m \sqrt{2 \frac{1-\cos \left(\frac{2 r-1}{m} \frac{\pi}{2}\right)}{2+\cos \left(\frac{2 r-1}{m} \frac{\pi}{2}\right)}}
\end{gathered}
$$

with $1<i, r<m$. The frequency overestimation given by $\bar{\omega}_{r} / \omega_{r}$ depends on the ratio $(2 r-1) / m$, where $2 r-1)$ is related to the wavelength $\lambda_{r}$ by the expression $\lambda_{r}=4 l /(2 r-1)$. This shows that the frequency error is a function of number of elements per wavelength.

\section{REFERENCES}

Allard J.-F. (1993). Propagation of sound in porous media : modeling sound absorbing materials, Chapman \& Hall

Atalla N., Panneton R. and Debergue P. (1996). "A mixed displacement-pressure formulation for Biot's poroelastic equations," J. Acoust. Soc. Am., 99, 2487

Nicolas Dauchez, Sohbi Sahraoui, Noureddine Atalla

The Journal of the Acoustical Society of America 
Atalla N., Panneton R. and Debergue P. (1998). "A mixed displacement-pressure formulation for poroelastic materials," J. Acoust. Soc. Am., 104, 1444-1452

Bardot A., Brouard B., Allard J.-F. (1996). "Frame decoupling at low frequency in thin porous layers saturated by air," J. Appl. Phys., 79, 8223-29

Biot, M.A. (1956). "The theory of propagation of elastic waves in a fluid-saturated porous solid. I. Low frequency range. II. Higher frequency range," J. Acoust. Soc. Am., 28, $168-191$

Blevins R.D. (1995). Formulas for natural frequency and mode shape, Krieger Publishing Company

Coyette J.P. and Wynendaele H. (1995). "A finite element model for predicting the acoustic transmission characteristics of layered structures," Proceedings of Inter-noise $1995,1279-1282$

Geradin M. and Rixen D. (1996). Théorie des vibrations - Application à la dynamique des structures, Masson

Johansen T.F., Allard J.-F. and Brouard B. (1995). "Finite element method for predicting the acoustical properties of porous samples," acta acoustica, 3, 487-491

Kang Y.J. and Bolton J.S. (1995). "Finite element modeling of isotropic elastic porous materials coupled with acoustical finite element," J. Acoust. Soc. Am., 98, 635-643

MacNeal R.H. (1994). Finite elements : their design and performance, Dekker

Panneton R. (1996). Modélisation numérique 3D par éléments finis des milieux 
poroélastiques, Ph.D. thesis, Université de Sherbrooke, Québec, Canada

Panneton R. and Atalla N. (1997). "An efficient finite element scheme for solving the three dimensional poroelasticity problem in acoustics," J. Acoust. Soc. Am., 101, 3287-3298

Petyt M. (1990). Introduction to finite element vibration analysis, Cambridge University Press 
Table 1. Characteristics of materials ( $\rho_{1}$ is the mass density of the solid phase).

\begin{tabular}{|l|c|c|c|c|c|}
\hline Material & $\phi$ & $\sigma$ & $\alpha_{\infty}$ & $\Lambda$ & $\Lambda^{\prime}$ \\
$\left(N m^{-4} s\right)$ & & $(\mu m)$ & $(\mu m)$ \\
\hline \hline $\mathrm{A}$ & 0.98 & 13500 & 1.7 & 80 & 160 \\
\hline $\mathrm{B}$ & 0.90 & 25000 & 7.8 & 2.5 & 2.5 \\
\hline $\mathrm{C}$ & 0.96 & 32000 & 1.7 & 90 & 165 \\
\hline
\end{tabular}

\begin{tabular}{|l|c|c|c|}
\hline Material & $\rho_{1}$ & $E$ & $\nu$ \\
$\left(k g ~ m^{-3}\right)$ & $(k P a)$ & \\
\hline \hline $\mathrm{A}$ & 30 & $270(1+\mathrm{j} 0.1)$ & 0.3 \\
\hline $\mathrm{B}$ & 30 & $800(1+\mathrm{j} 0.25)$ & 0.4 \\
\hline $\mathrm{C}$ & 30 & $845(1+\mathrm{j} 0.1)$ & 0.3 \\
\hline
\end{tabular}


Table 2. Convergence of first bending mode frequency versus number of elements in lateral and thickness directions, for a clamped elastic layer $(28 \mathrm{~cm} \times 35 \mathrm{~cm} \times 3 \mathrm{~cm})$ equivalent to material $\mathrm{A}$.

\begin{tabular}{|c|c|c|c|c|c|}
\hline \multirow{2}{*}{\multicolumn{2}{|c|}{$\Delta f(\%)$}} & \multicolumn{4}{|c|}{ Lateral directions } \\
\hline & & 3 & 6 & 12 & 24 \\
\hline \multirow{4}{*}{ 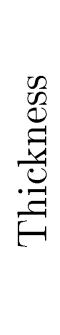 } & 1 & 46 & 16 & 10 & 9 \\
\hline & 2 & 45 & 11 & 5 & 3 \\
\hline & 4 & 42 & 9 & 3 & 1 \\
\hline & 8 & 40 & 8 & 2 & 0 \\
\hline
\end{tabular}


Table 3. Convergence of the first bending mode of a clamped porous layer $(28 \mathrm{~cm} \times 35$ $\mathrm{cm} \times 3 \mathrm{~cm}$; material A) versus number of elements in lateral and thickness directions :

a) frequency; b) dissipated power by viscous effect.

\begin{tabular}{|c|c|c|c|c|}
\hline \multirow{2}{*}{\multicolumn{2}{|c|}{$\Delta f(\%)$}} & \multicolumn{3}{|c|}{ Lateral directions } \\
\hline & & 6 & 12 & 24 \\
\hline \multirow{4}{*}{ 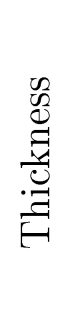 } & 1 & 40 & 15 & 7 \\
\hline & 2 & 36 & 10 & 3 \\
\hline & 4 & 35 & 8 & 1 \\
\hline & 8 & 34 & 7 & 0 \\
\hline
\end{tabular}

\begin{tabular}{|c|c|c|c|c|}
\hline \multirow{2}{*}{\multicolumn{2}{|c|}{$\Delta D_{c p}(\%)$}} & \multicolumn{3}{|c|}{ Lateral directions } \\
\hline & & 6 & 12 & 24 \\
\hline \multirow{4}{*}{ 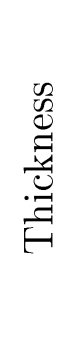 } & 1 & 89 & 97 & 104 \\
\hline & 2 & 84 & 94 & 97 \\
\hline & 4 & 64 & 49 & 38 \\
\hline & 8 & 54 & 17 & 0 \\
\hline
\end{tabular}




\section{Figure captions}

Figure 1. Porous layer of thickness $l$, bonded to a rigid impervious wall.

Figure 2. Relative contributions $\left|C_{s w / f}\right|$ and $\left|C_{f w / s}\right|$ at the incident face $(x=-l)$, for a $10 \mathrm{~cm}$ thick layer of material A (table 1).

Figure 3. Convergence rate of 1-D linear elastic elements.

Figure 4. Mesh of a porous layer : a) imposed transverse displacement, b) imposed longitudinal displacement, c) normal incidence acoustical wave.

Figure 5. Shear excited porous column (material C) : a) velocity of the free face; b) convergence rate.

Figure 6. Mechanically excited porous column : a) free face velocity (material C); b) convergence rate for several materials (A and B) and types of element. H8 is related to hexaedric element (brick) and P6 to pentaedric element (straight prism).

Figure 7. Acoustically excited $7.5 \mathrm{~cm}$ thick column (material B) : a) velocity of the excited face; b) convergence rate.

Figure 8. Acoustically excited $7.5 \mathrm{~cm}$ thick column (material B) : a) $\left|C_{s w / f}\right|$ and $\left|C_{f w / s}\right|$ ratio at the incident face, for a $7.5 \mathrm{~cm}$ thick layer; b) Velocity of the fluid phase at the free face for severals meshes, compared to the converged solution.

Figure 9. Acoustically excited porous column : a) displacement amplitude along axis $x$ of the two phases at first resonance of the solid phase $(809 \mathrm{~Hz})$. b) example of an irregular mesh.

Figure 10. Clamped poroelastic layer excited by a normal incidence acoustical wave.

Figure 11. Biot wavelengths for materials A, B and C. 
Figure 1.

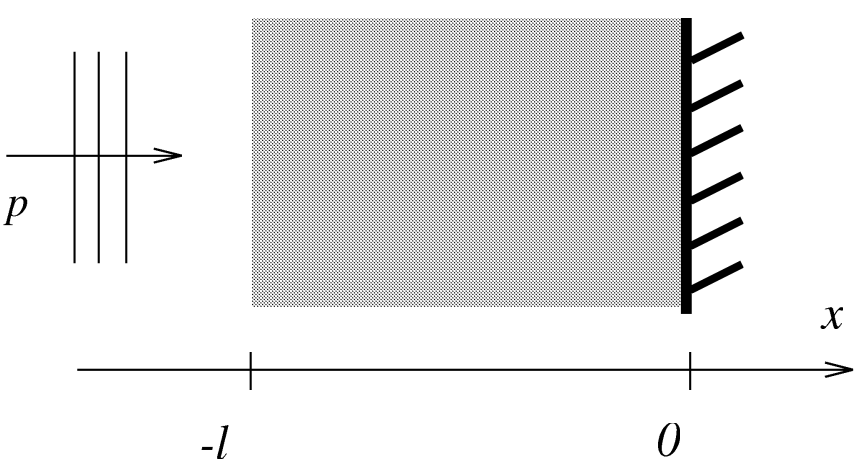

Nicolas Dauchez, Sohbi Sahraoui, Noureddine Atalla The Journal of the Acoustical Society of America 
Figure 2.

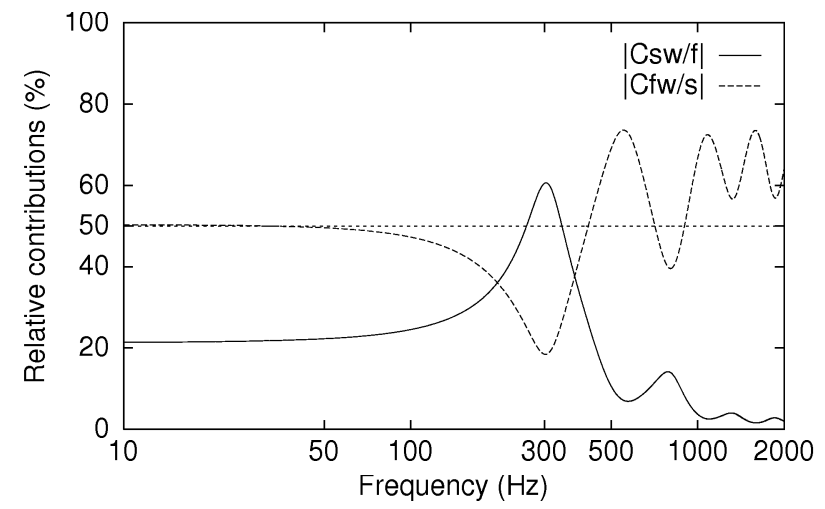

Nicolas Dauchez, Sohbi Sahraoui, Noureddine Atalla The Journal of the Acoustical Society of America 
Figure 3.

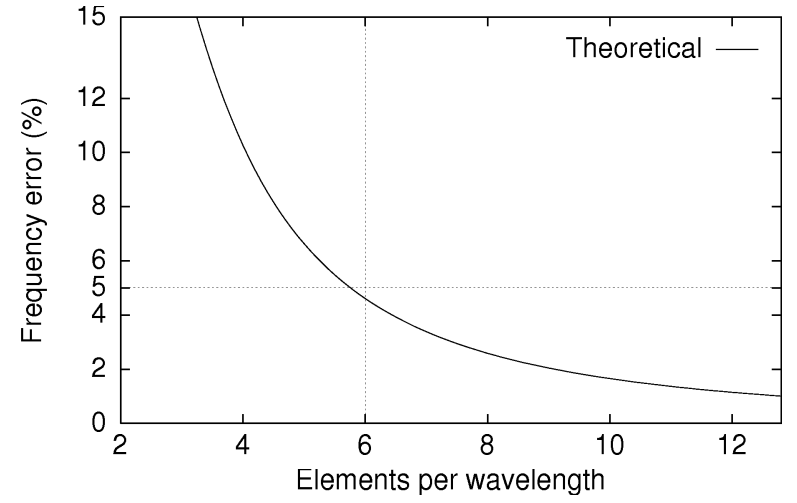

Nicolas Dauchez, Sohbi Sahraoui, Noureddine Atalla The Journal of the Acoustical Society of America 
Figure 4.

(a)

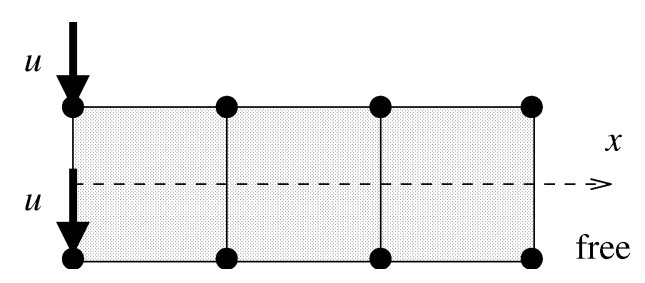

face

(b)

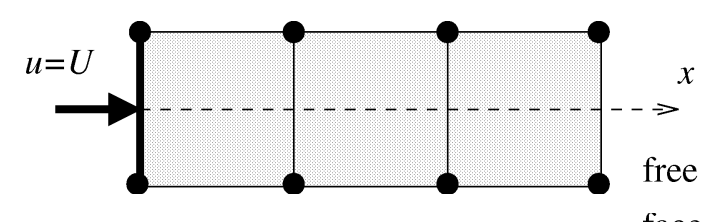

(c)

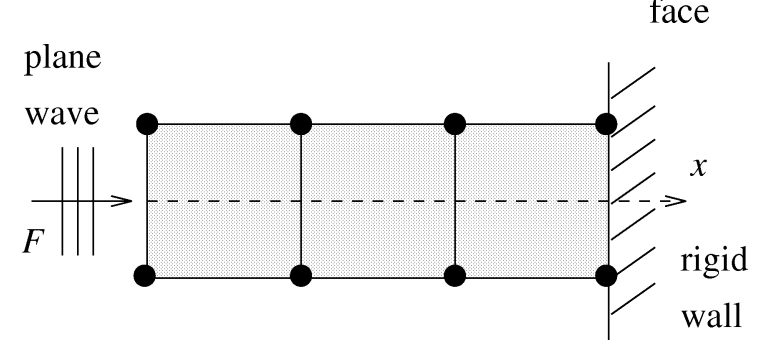


Figure 5.
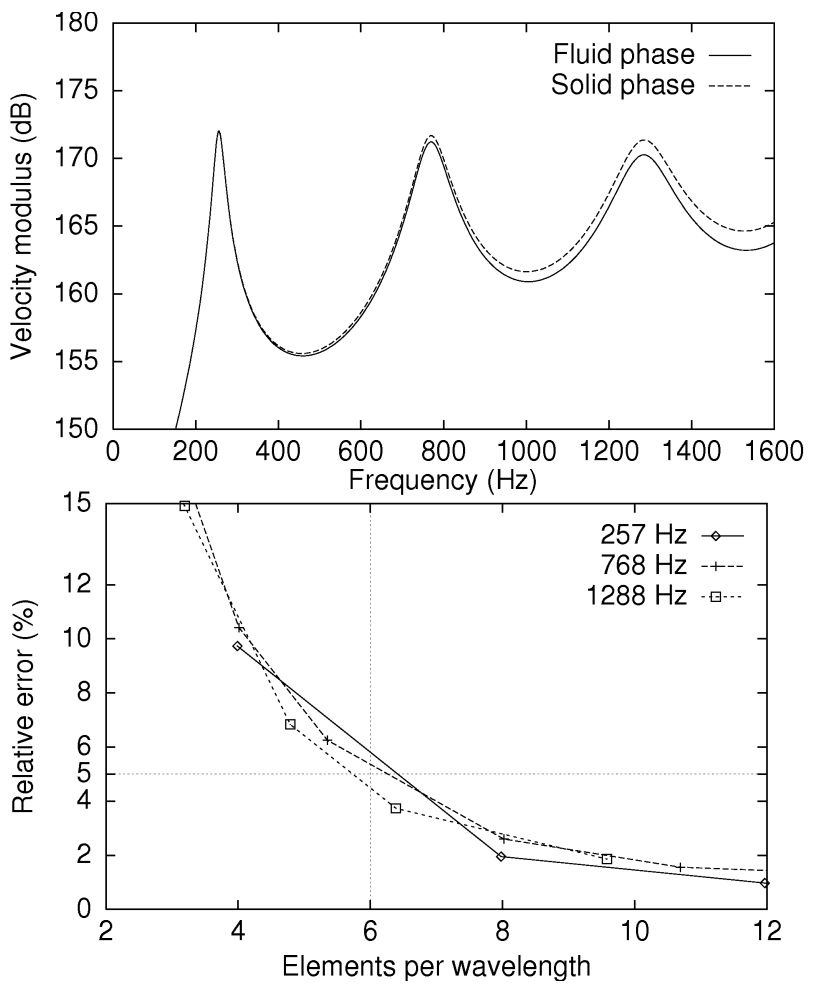

Nicolas Dauchez, Sohbi Sahraoui, Noureddine Atalla The Journal of the Acoustical Society of America 
Figure 6.
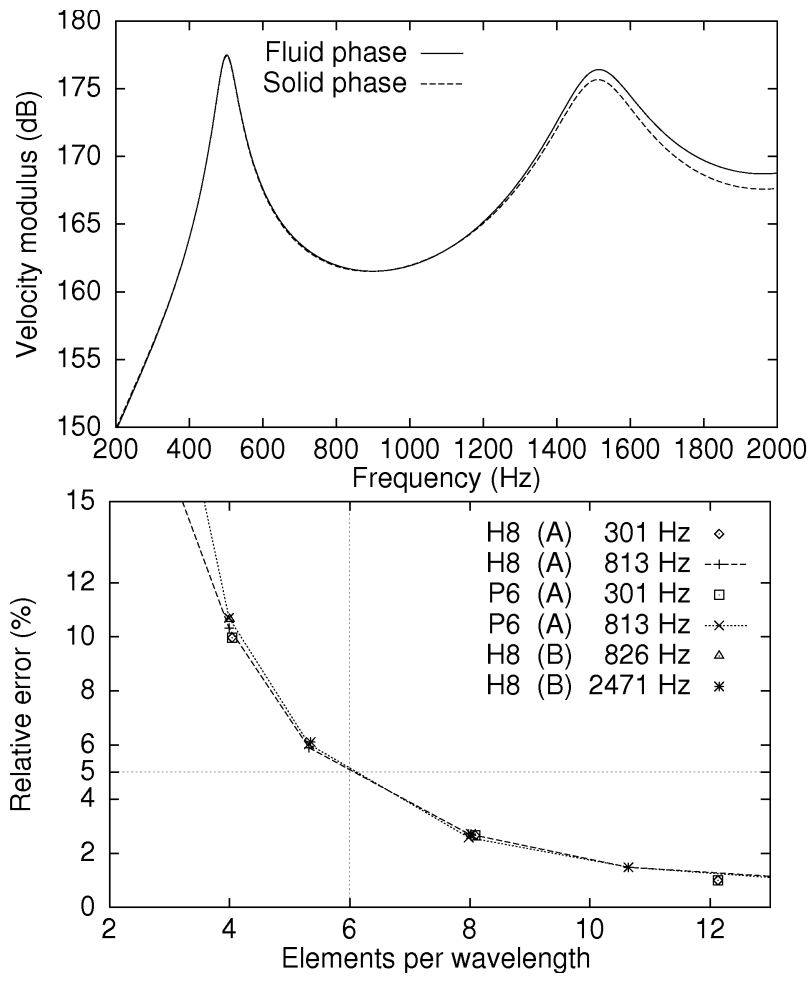

Nicolas Dauchez, Sohbi Sahraoui, Noureddine Atalla The Journal of the Acoustical Society of America 
Figure 7.
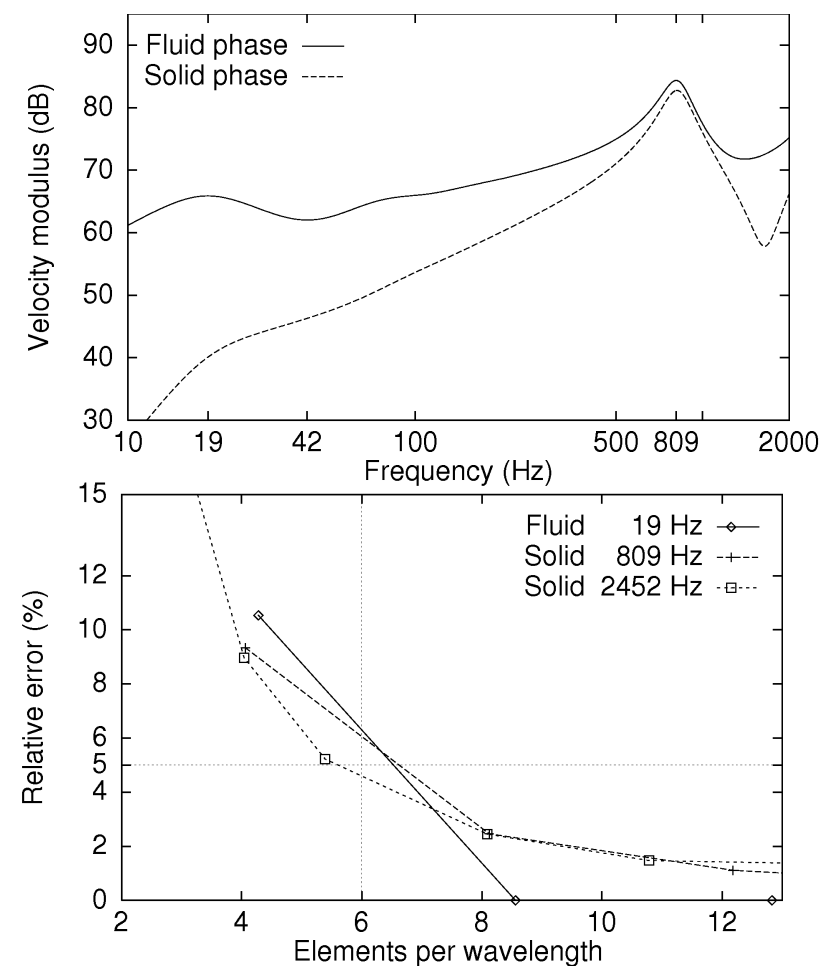

Nicolas Dauchez, Sohbi Sahraoui, Noureddine Atalla The Journal of the Acoustical Society of America 
Figure 8.
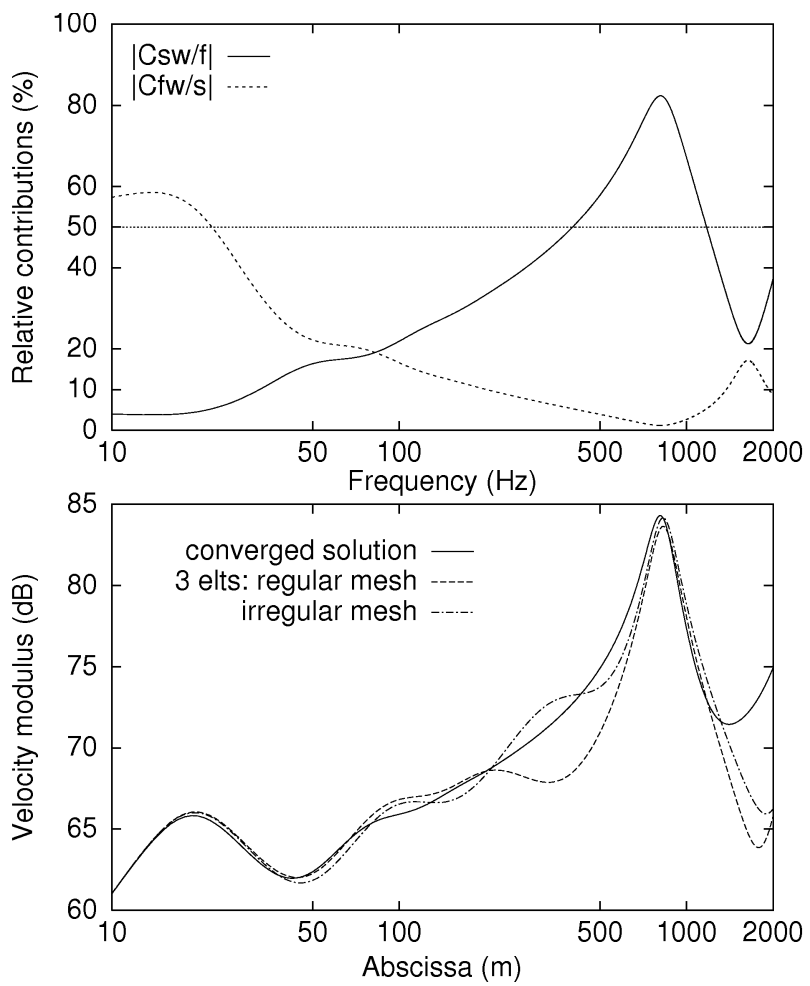

Nicolas Dauchez, Sohbi Sahraoui, Noureddine Atalla The Journal of the Acoustical Society of America 
Figure 9.
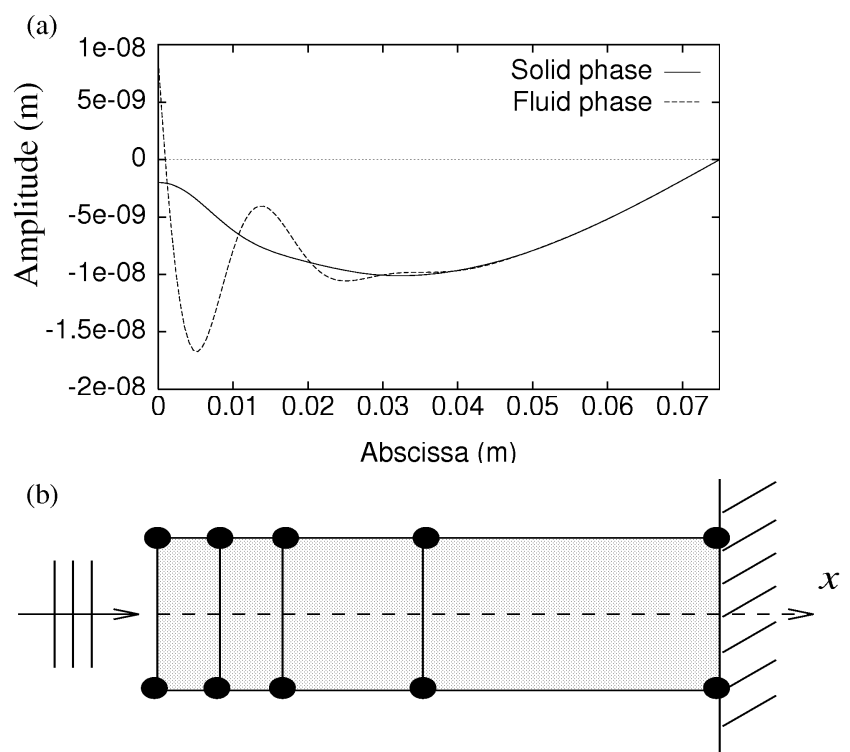

Nicolas Dauchez, Sohbi Sahraoui, Noureddine Atalla The Journal of the Acoustical Society of America 
Figure 10.

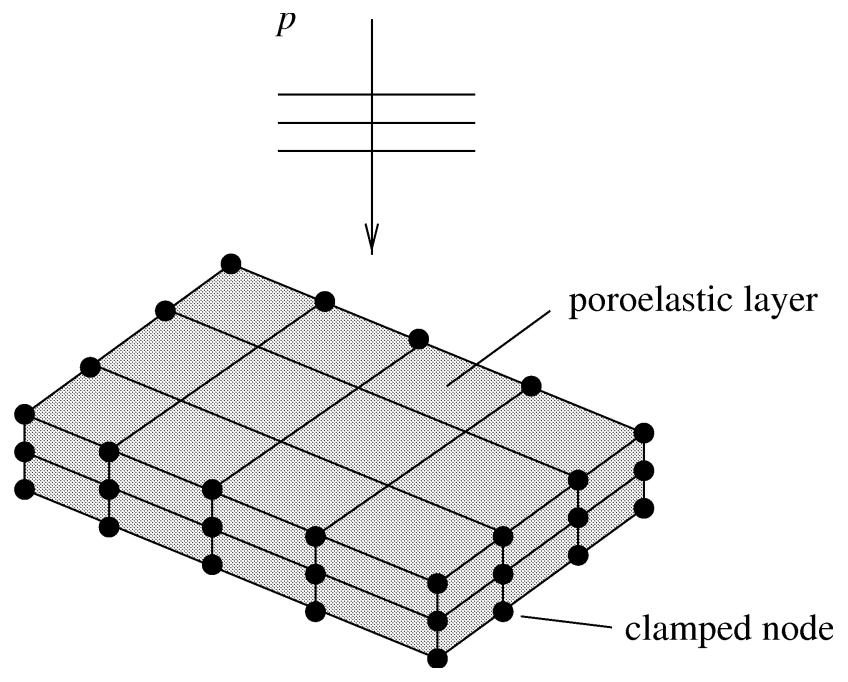

Nicolas Dauchez, Sohbi Sahraoui, Noureddine Atalla The Journal of the Acoustical Society of America 
Figure 11.
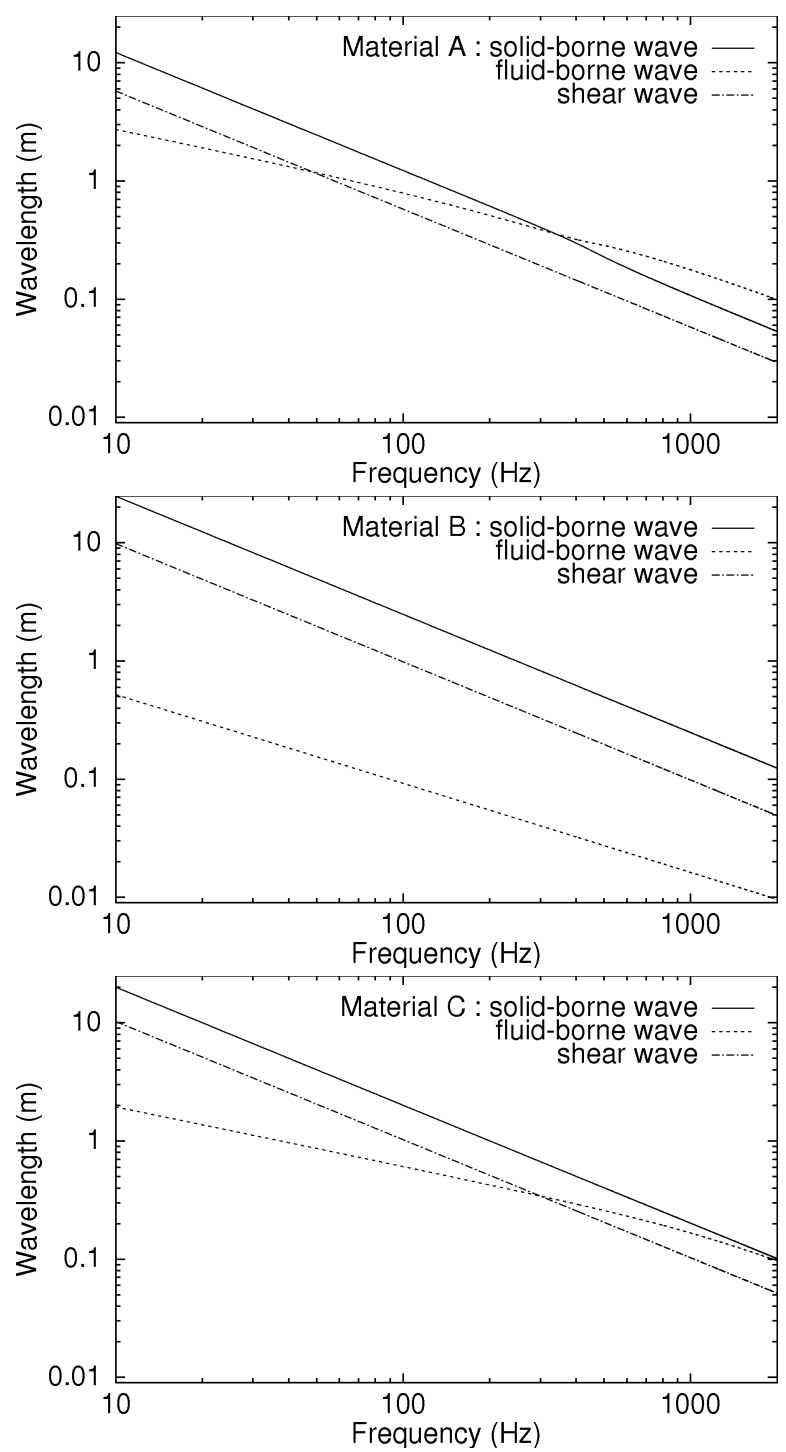

Nicolas Dauchez, Sohbi Sahraoui, Noureddine Atalla The Journal of the Acoustical Society of America 